\title{
Penetrating 3-D Imaging at 4- and 25-m Range Using a Submillimeter-Wave Radar
}

Ken B. Cooper, Member, IEEE, Robert J. Dengler, Member, IEEE, Nuria Llombart, Member, IEEE, Tomas Bryllert, Goutam Chattopadhyay, Senior Member, IEEE, Erich Schlecht, Member, IEEE, John Gill, Choonsup Lee, Anders Skalare, Member, IEEE, Imran Mehdi, Senior Member, IEEE, and Peter H. Siegel, Fellow, IEEE

\begin{abstract}
We show experimentally that a high-resolution imaging radar operating at $576-605 \mathrm{GHz}$ is capable of detecting weapons concealed by clothing at standoff ranges of 4-25 m. We also demonstrate the critical advantage of 3-D image reconstruction for visualizing hidden objects using active-illumination coherent terahertz imaging. The present system can image a torso with $<1 \mathrm{~cm}$ resolution at $4 \mathrm{~m}$ standoff in about five minutes. Greater standoff distances and much higher frame rates should be achievable by capitalizing on the bandwidth, output power, and compactness of solid state Schottky-diode based terahertz mixers and multiplied sources.
\end{abstract}

Index Terms-Millimeter waves, submillimeter-wavelength imaging, terahertz radar, terharertz (THz).

\section{INTRODUCTION}

$\mathbf{N}$ UMEROUS commercial technologies, from trace chemical sniffers to X-ray imagers, can detect weapons or contraband concealed on persons, but in almost all cases these approaches require the sensor and the target to be in close proximity. For situations that call for remote detection, such as when hidden explosives may be detonated or where clandestine surveillance is warranted, concealed weapons detection is at best extremely difficult to accomplish.

One technique that shows great promise for achieving through-garment imaging at standoff ranges from 5 to $100 \mathrm{~m}$ is active submillimeter-wave imaging using coherent heterodyne detection (mixers). Between $100 \mathrm{GHz}$ and $1 \mathrm{THz}$ most clothing is reasonably transmissive [1], while at the same time high image resolution can be achieved with a compact aperture. For example, for a standoff range $R=50 \mathrm{~m}$, an antenna diameter $D=1 \mathrm{~m}$, and an operating wavelength $\lambda=0.5 \mathrm{~mm}$ $(600 \mathrm{GHz})$, the two-way diffraction-limited image resolution is about $2 \mathrm{~cm}$, sufficient for many threat scenarios.

Manuscript received April 10, 2008; revised September 10, 2008. First published November 18, 2008; current version published December 05, 2008. This work was supported by the National Aeronautics and Space Administration (NASA) under a contract, by the Division of Biology, California Institute of Technology, by the Naval Explosive Ordnance Disposal Technology Division with funding provided by the Department of Defense (DoD) Physical Security Equipment Action Group (PSEAG).

K. B. Cooper, R. J. Dengler, N. Llombart, T. Bryllert, G. Chattopadhyay, E. Schlecht, J. Gill, C. Lee, A. Skalare, and I. Mehdi are with the Jet Propulsion Laboratory, California Institute of Technology, Pasadena, CA 91109 USA.

P. H. Siegel is with the Jet Propulsion Laboratory, California Institute of Technology, Pasadena, CA 91109 USA, and also with the Department of Biology, California Institute of Technology, Pasadena, CA 91125 USA (e-mail: phs@ caltech.edu).

Color versions of one or more of the figures in this paper are available online at http://ieeexplore.ieee.org.

Digital Object Identifier 10.1109/TMTT.2008.2007081
Excellent sources and sensors are also now available at terahertz frequencies. For example, the Jet Propulsion Laboratory's best all-solid-state submillimeter-wave Schottky diode-based sources produce $>2 \mathrm{~mW}$ of coherent output power while Schottky mixers exhibit a 2000 K DSB mixer noise temperature around $600 \mathrm{GHz}$ [2]-[4]. Even derating these parameters by a factor of two to $1 \mathrm{~mW}$ and $4000 \mathrm{~K} \mathrm{DSB}$, we can derive excellent signal-to-noise for a radar application. For instance, taking an additional loss of $10 \mathrm{~dB}$ from atmospheric absorption, optics, and RF circuitry, the total noise temperature of a submillimeter imaging system using these components will be $40,000 \mathrm{~K}$. Assuming that a target has a backscattering coefficient of $-20 \mathrm{~dB}$ (i.e., isotropically reflecting $1 \%$ of the incident radiation), and that the integration time is $10 \mathrm{~ms}$, the signal-to-noise ratio (SNR) will be $\sim 67 \mathrm{~dB}$ according to a conventional radar analysis [5]. With the ability to penetrate clothing, the potential for $\mathrm{cm}$-scale image resolution, and SNR in excess of $10^{6}$, it is no wonder that active submillimeter-wavelength imaging has attracted great interest for standoff weapons detection. But is this substantial sensitivity and good resolution sufficient to reliably detect concealed objects?

In this paper we show that it is not sufficient because in real-life scenarios a single-pixel coherent image will typically exhibit very poor contrast between a concealed object and the surrounding clothing and skin-even for metallic objects such as guns. Instead, the challenge of active terahertz detection of concealed objects involves extracting signals from scene clutter rather than from noise. We have addressed this problem by implementing for the first time an ultra-broadband submillimeter-wave radar that can map a person at the sub-cm scale in three dimensions. Using this technique, we are able to generate high-resolution images and remove the clutter signals to reveal hidden objects at standoff ranges of 4 and $25 \mathrm{~m}$. This work represents a significant improvement over that described in [6], where only a brightly reflective concealed object could be detected at $4 \mathrm{~m}$ standoff, and where the image resolution was inferior in all dimensions. We also show here how the system can be readily scaled to larger standoff ranges, but increasing its speed to near-real-time frame rates will require a substantial effort to develop a multi-pixel imaging radar array.

\section{High-Resolution FrequenCy-Modulated CONTINUOUS-WAVE (FMCW) RADAR}

The block diagram of an earlier version of the $600 \mathrm{GHz}$ FMCW radar has already been reported in [6], and it is reproduced here in Fig. 1(a). The main change to the system 


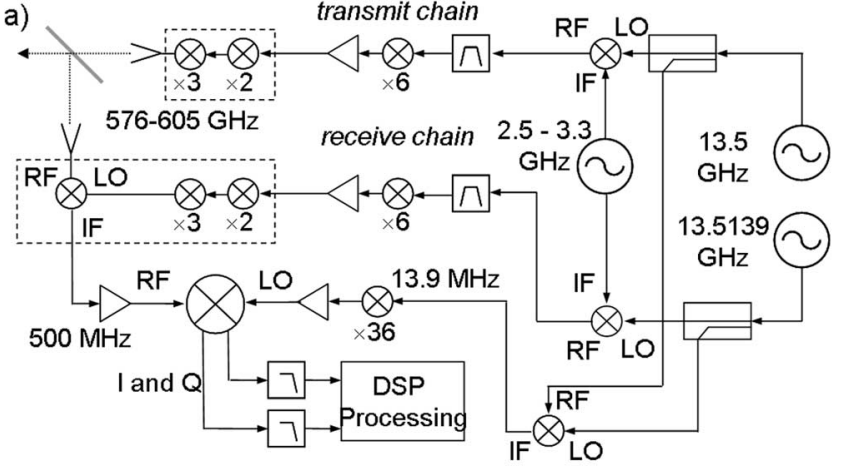

b) $40 \mathrm{~cm}$ reflector lens

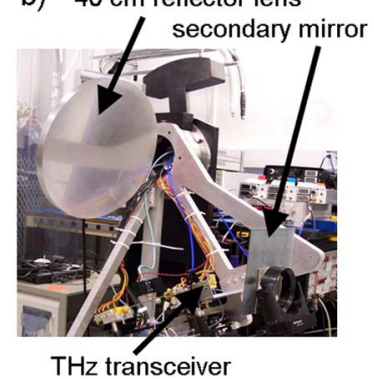

c)

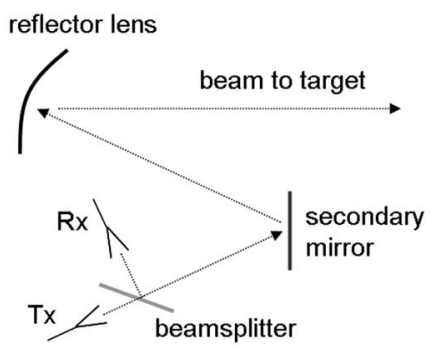

Fig. 1. (a) Block diagram of $600 \mathrm{GHz}$ radar imager. The transmit signal and the receiver $\mathrm{LO}$ are generated by multiplying microwave sources by a factor of 36. A $2.5-3.3 \mathrm{GHz}$ chirp, generated by a DDS/PLL synthesizer, results in a final FMCW signal with a $28.8 \mathrm{GHz}$ bandwidth. The submillimeter-wave multipliers and mixer, designed and built at the Jet Propulsion Laboratory, are enclosed by dashed boxes. (b) and (c) Radar photograph and optics schematic.

electronics is that a hybrid direct digital synthesis/phase-lock loop (DSS/PLL) synthesizer is used to generate the chirped signal, instead of a YIG synthesizer. The DSS/PLL is typically ramped between 2.5 and $3.3 \mathrm{GHz}$ in $12.5 \mathrm{~ms}$. After upconversion onto a synthesized $13.5 \mathrm{GHz}$ signal, followed by $\times 36$ frequency multiplication, this results in a transmitted 576-604.8 $\mathrm{GHz}$ chirp with a rate $K=2.3 \mathrm{MHz} / \mu$ s. This chirp waveform has a bandwidth of $604.8-576=28.8 \mathrm{GHz}$. A radar's range resolution is inversely proportional to its bandwidth according to the formula $\Delta r=c / 2 \Delta F$, where $\Delta r$ is the resolution, $c$ is the speed of light and $\Delta F$ is the chirp radar bandwidth [5]. Therefore the system described here can theoretically attain a range resolution of about $0.5 \mathrm{~cm}$, roughly a factor of 2 improvement over the previous system. Also, the $\times 4$ speed-up in the attainable repetition frequency permits images to be acquired more quickly. The use of a DDS-based synthesizer for FMCW radar, rather than a YIG or VCO chirper, is usually motivated by the high chirp linearity needed for high range resolution. However, our $600 \mathrm{GHz}$ radar exhibits intrinsic nonlinearities from its submillimeter-wave components that are at least as large as what a YIG or VCO would produce. Nonetheless we found that the stability of the ramp waveform generated by the DDS/PLL hybrid was superior to that of all-analog sweepers, and waveform stability is the key figure of merit that permits us to attain close to the bandwidth-limited range resolution of $0.5 \mathrm{~cm}$ by using a digital phase- and amplitude-compensation algorithm for ramp nonlinearities [7].

Another improvement made to the imaging radar is that the beam focusing and scanning is now accomplished by an
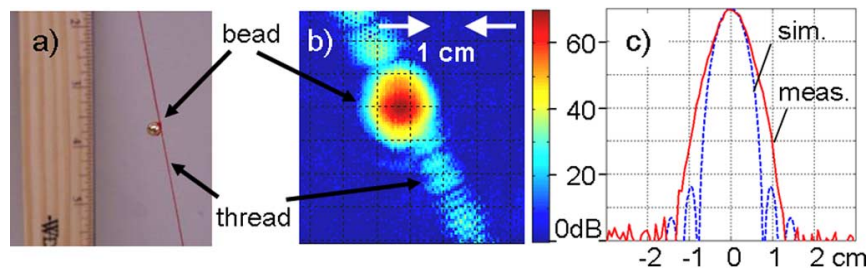

Fig. 2. (a) $3 \mathrm{~mm}$ gold-plated bead suspended by a thread. (b) $600 \mathrm{GHz}$ scanned radar image of the bead at $4 \mathrm{~m}$ standoff. Even the cotton thread is detected by the radar. (c) Measured horizontal cross-section of the bead's return power through the bead center (solid) and the simulated two-way beam pattern of the main antenna (dashed).

aluminum off-axis ellipsoidal reflector mounted on a two-axis rotation stage. Using a precision-machined reflector fabricated by Custom Microwave Inc., rather than a refractive Teflon plano-convex lens, boosts the optical efficiency by approximately $8 \mathrm{~dB}$ due to lower absorption loss and the elimination of reflection loss from the $\sim 2 \mathrm{~cm}$ thick dielectric lens [8]. Clutter resulting from the lens backscattering was also eliminated. Fig. 1(b) shows a photograph of the $600 \mathrm{GHz}$ imaging radar stage with a $40 \mathrm{~cm}$ diameter mirror installed. A beam diagram is shown in Fig. 1(c). Upon leaving the transceiver the first null beam width is $28^{\circ}$, and a secondary mirror deflects the beam to the primary reflector for focusing on a target.

The ellipsoidal reflector was designed using the physical optics simulation software GRASP (TICRA, Denmark) to achieve a simulated diffraction-limited half-power beam width of $6 \mathrm{~mm}$. Owing to the two-way nature of the transceiver, the antenna pattern is squared to yield an effective $3 \mathrm{~dB}$ imaging beam width of $4 \mathrm{~mm}$. Instead of performing a calibrated measurement of the radar's antenna pattern, we assessed its beam quality by imaging a $3 \mathrm{~mm}$ diameter gold-plated bead suspended by a single cotton threat at $4 \mathrm{~m}$ standoff. Fig. 2(a) shows a photograph of the scene along with a ruler that was not present during the scan, and Fig. 2(b) shows the range-gated received power of the bead on a logarithmic scale. Each of the approximately 10000 pixels in this radar image was obtained using a $25 \mathrm{~ms}$ chirp and integration time, and the peak power received from the bead is about $60 \mathrm{~dB}$ above the receiver's intrinsic noise floor and $50 \mathrm{~dB}$ above the phase-noise-limited noise floor. Note that in Fig. 2(a) even a single thread is detectable with an SNR exceeding $20 \mathrm{~dB}$ in some places.

In Fig. 2(c), a cross-section of the measured bead signal in Fig. 2(b) is shown along with the two-way (squared) antenna beam pattern calculated by GRASP, and the match between experiment and theory is excellent down to about $15 \mathrm{~dB}$. Below that, the discrepancies between the curves may be due to effects such as misaligned optics or the use of a target whose cross-section is not small compared to the beam. Regardless, the results of Fig. 2 prove that the $600 \mathrm{GHz}$ radar imager is capable of detecting targets with very high sensitivity and with sub-cm cross-range resolution.

While cross-range resolution is a key figure of merit for a two-dimensional imager, in a 3-D radar imager the range resolution is also critical. To assess the range resolution capabilities of the $600 \mathrm{GHz}$ imaging radar, a target was used with rele- 


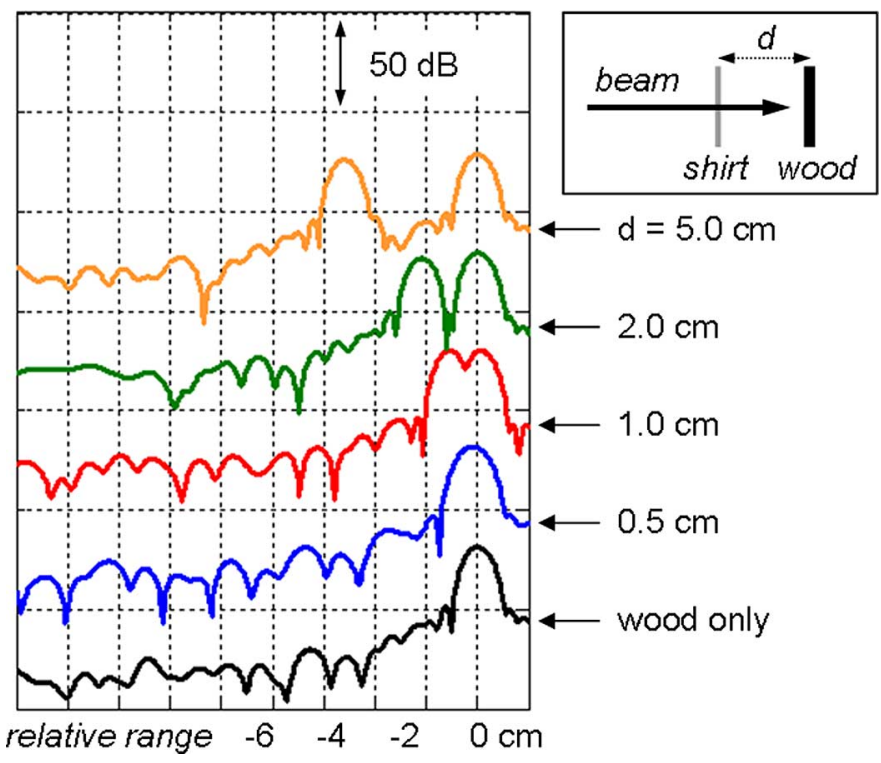

Fig. 3. Range-compressed radar returns (power spectra) of a stretched shirt in front of a flat wooden board at various separations. Two distinct peaks occur once the shirt-wood gap reaches about $1 \mathrm{~cm}$, the practical range resolution of the $600 \mathrm{GHz}$ radar.

vance to through-garment imaging. Fabric from a cotton T-shirt was stretched flat and positioned a short distance in front of a wooden board. The shirt and wood were placed approximately perpendicular to the radar beam at a $4 \mathrm{~m}$ standoff range. While the beam can penetrate the shirt very easily, the 0.5 -in thick wood is opaque at $600 \mathrm{GHz}$, with a reflected signal only being detectable from its front surface. We define the imager's range resolution for this scenario as the minimum distance between the shirt and wood such that the radar can still distinguish between the two.

Fig. 3 shows the range-compressed power spectra obtained with the $600 \mathrm{GHz}$ radar for four different shirt-wood separations between 0.5 and $5.0 \mathrm{~cm}$, in addition to the wood-only spectrum. These data indicate that at $0.5 \mathrm{~cm}$ separation, which is the theoretical bandwidth-limited range resolution of the radar, only one peak is evident. By $1 \mathrm{~cm}$ separation, two peaks can clearly be discerned with a contrast of about $8 \mathrm{~dB}$. Thus the practical range resolution of the $600 \mathrm{GHz}$ radar is around $1 \mathrm{~cm}$, about twice the $28.8 \mathrm{GHz}$ bandwidth-limited value. This reduction in resolution results from the use of a Hann window in the power spectrum calculation, which increases the point-target spectral width by about $60 \%$, and residual uncompensated FMCW ramp nonlinearities.

\section{CONCEALED WEAPONS DETECTION}

The added value of high-resolution ranging to active heterodyne submillimeter-wave imaging is conveyed by comparing the detectability of a concealed gun with varying degrees of range resolution. Fig. 4(a) shows a photograph of the imaging target: a person with a 0.45 caliber handgun replica concealed underneath his shirt at $4 \mathrm{~m}$ standoff. The imagery displayed in Fig. 4(b) and (c) were generated from the same scan of the target by the $600 \mathrm{GHz}$ imaging radar. These data were acquired by raster-scanning the radar beam with the fast direction along the
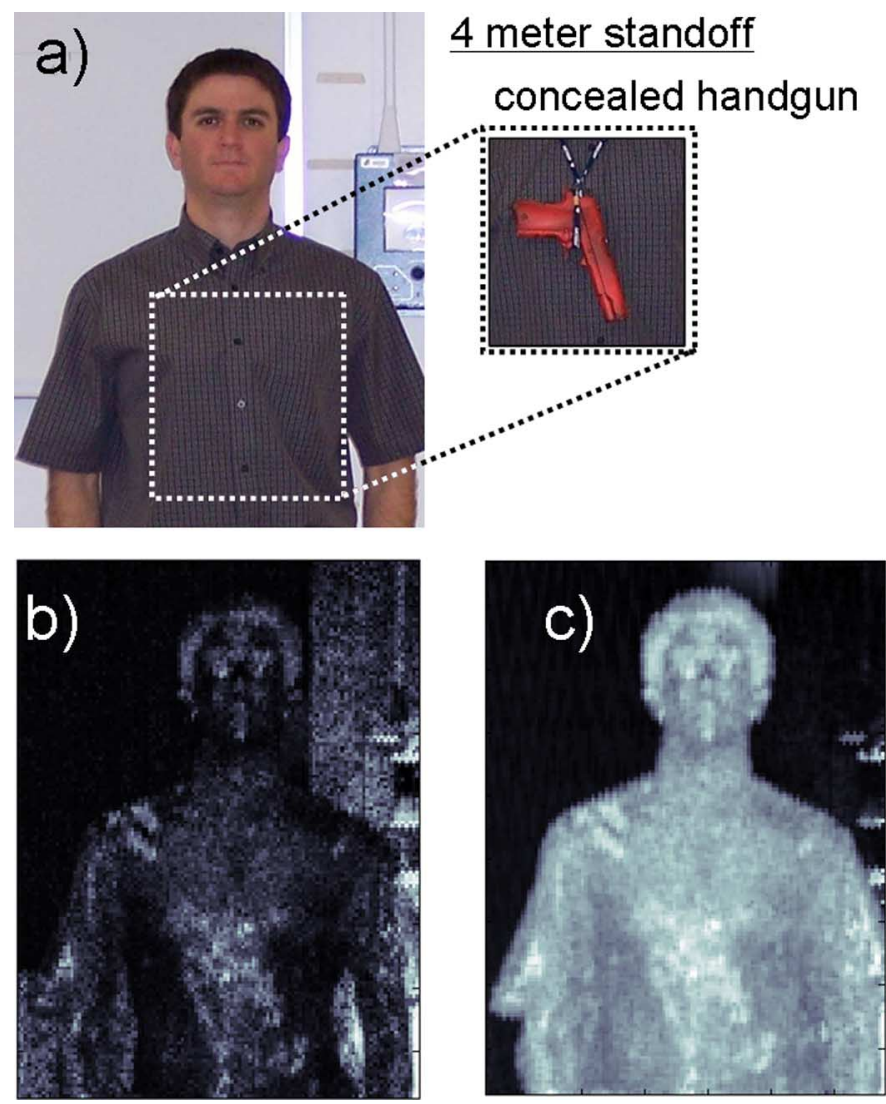

Fig. 4. (a) Target scenario, where a concealed metallic gun replica is hung around the subject's neck and beneath his shirt at $4 \mathrm{~m}$ standoff range. (b) Totalpower image of the received intensity, on a logarithmic scale, covering the entire final IF bandwidth of $96 \mathrm{kHz}$ (range swath $6.25 \mathrm{~m}$ ). (c) Image of the received power (log scale) after course filtering over a range swath of $50 \mathrm{~cm}$ centered on the subject. Though the image is greatly improved, the concealed gun remains hidden because of shirt and skin clutter signals.

vertical (elevation) axis, resulting in $17 \times 10^{3}$ FMCW radar pulses collected, and giving a pixel spacing of $0.6 \mathrm{~cm}$ for the $151 \times 113$ pixel image. Each chirp waveform $(28.8 \mathrm{GHz}$ bandwidth) was acquired in $12.5 \mathrm{~ms}$. With the added delays from signal processing and mechanical stage motion, the total image acquisition time was about 6 minutes.

Fig. 4(b) shows a grayscale scene image of the total power (on a log scale) collected by the radar at each pixel. The power was calculated by directly summing and squaring the $I$ and $Q$ signal voltages sampled at $316 \mathrm{kHz}$ during each $12.5 \mathrm{~ms}$ dwell of the FMCW waveform. Prior to analog-to-digital conversion, the $I$ and $Q$ channels were low-pass filtered at $96 \mathrm{kHz}$, which excludes any spurious target signals that are beyond $6.25 \mathrm{~m}$. (In FMCW radar, range $R$ is related to IF frequency $f_{\mathrm{IF}}$ by the equation $f_{\mathrm{IF}}=2 K R / c$, with $K$ the chirp rate and $c$ the speed of light.) As a result, the total-power image of Fig. 4(b) includes contributions from the targeted person, clutter from the wall behind him, and the close-range clutter caused by backscattering of the signal from the scanning stage and optics.

These strong clutter sources make the reflected-power image of Fig. 4(b) difficult to interpret. For example, the subject's neck is virtually invisible, and his torso is poorly contrasted to the background. These observations are quite similar to those reported in [9], where a person was imaged using an active but 
non-ranging $640 \mathrm{GHz}$ imager. However, unlike in [9], the concealed handgun in Fig. 4(b) cannot be seen, aside from a slightly higher region of reflectivity near the chest. This region of increased reflection is not well-resolved spatially and is not significantly brighter than, for example, the shirt/skin-only reflections near the subject's right shoulder. This may be surprising because the handgun surface is almost completely metallic, and hence nearly $100 \%$ reflective, while skin and clothes typically reflect only a few percent of the incident energy. However, unless a smooth metallic surface is oriented almost exactly normal to the beam direction, its backscattering coefficient will be extremely small [5]. Separate tests we performed on flat, unpolished aluminum indicate that, for the geometry of our experiment, the backscattering coefficient measured by our radar drops by more than $60 \mathrm{~dB}$ after only a $2^{\circ}$ rotation away from normal incidence. Thus even for a metallic object such as a handgun, a near-normal angle of incidence is required to form a discernable image when using power-only measurements.

Fig. 4(c) shows another log-power image from the same data set, but with additional digital filtering covering a bandwidth of $8 \mathrm{kHz}$ and corresponding to a range swath of $50 \mathrm{~cm}$ centered on the subject's body. This filtering excludes the majority of the clutter from the far wall and the close-in backscattering, and as a result the image is significantly clearer than Fig. 4(b). For example, the full outline of the subject is visible, including low-reflectivity regions such as around the neck and much of the face. However, in Fig. 4(c) there is little indication that the subject is concealing a handgun because even with the background clutter reduction, the reflected power contrast across the target's body is still dominated by variations in the angle of incidence between the beam and the target. The consistent lack of contrast in reflected power from concealed guns or other objects we have tested leads us to conclude that active single-pixel coherent submillimeter-wave systems will exhibit limited detection ability when operated in a power-only imagery modality.

As an alternative imaging approach, in Fig. 5 we demonstrate how by neglecting reflectivity contrast and instead utilizing the high range resolution capability of the radar, excellent through-clothes imagery can be generated from the same raw data set used in Fig. 4. The images in Fig. 5(b) and (c) were made using a signal and image processing algorithm consisting of the following steps: First, for each pixel of the image, the digitized I/Q radar return signal is phase- and amplitude-compensated to correct for chirp nonlinearities and then range-compressed using the fast Fourier transform (FFT). This generates a power spectrum with a frequency axis corresponding to absolute range to the target. An example is shown in Fig. 5(a), which shows the spectrum from a single pixel located about $12 \mathrm{~cm}$ below the gun handle (see inset). Second, a peak-finding algorithm identifies potential targets based on their signal strength and peak width exceeding certain thresholds, typically $20-30 \mathrm{~dB}$ above the noise floor and 2-3 range bins, respectively.

The third and most important step is to identify the nearest and farthest peaks for each pixel of the image, i.e., the ranges to the first and the last object that the beam is scattered from during its trajectory. For imaging people, the first object the beam usually encounters will be either an outer layer of clothing surface or an exposed portion of the body. The last object encountered,

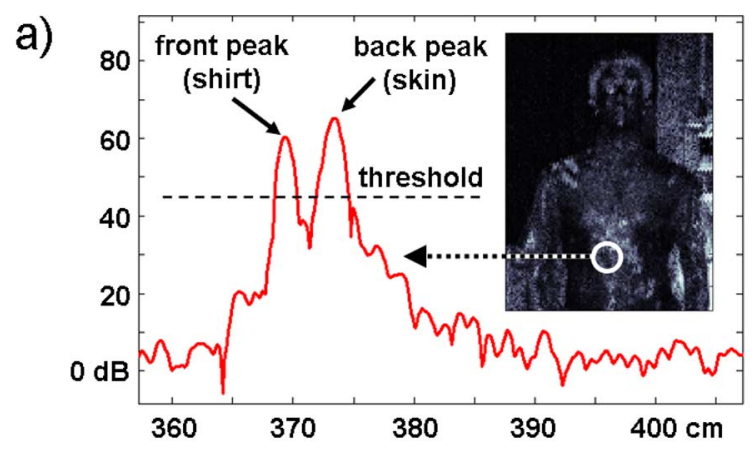
b)
front surface
C) back surface

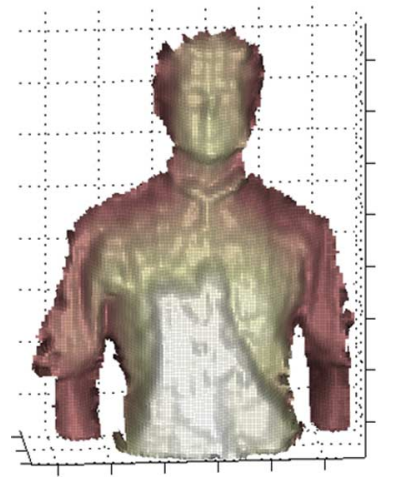

Fig. 5. (a) Power spectrum of individual pixel from dataset of Fig. 4. The pixel location is indicated by a circle in the inset total-power image. Two peaks exceeding threshold come from the subject's shirt and body surface. (b) and (c) Full 3-D reconstructions, after some additional smoothing, of the front and back surface ranges consisting of the identified front and back peaks for each pixel. The concealed handgun is visible as a bulge in the back surface.

provided the clothing is sufficiently transmissive at $600 \mathrm{GHz}$, will be either the person's body (through which terahertz radiation does not propagate) or an opaque concealed object. For the pixel highlighted in Fig. 5(a), the locations of the front and the back peaks are indicated by arrows, and these correspond to the subject's shirt and skin. As already demonstrated in Fig. 3, separate layers as narrow as $1 \mathrm{~cm}$ can be detected with the high-resolution $600 \mathrm{GHz}$ imaging radar. In this case, the shirt/skin gap is about $4 \mathrm{~cm}$ and is easily resolved. Similar spectra are obtained when the beam is aimed toward the gun, except in that case the back peak's range corresponds to the gun surface instead of the skin surface.

The fourth and final step of the algorithm is to assemble the front and back peak ranges, collected over the entire image, into 3-D "front surface" and "back surface" reconstructions. This step includes a smoothing routine to remove the occasional spurious dip or spike in the surface reconstruction that usually comes from misidentifying the front and back peaks. For example, multiple reflections of the beam (i.e., multipath signals) can generate a nonphysical peak at a range more distant than the skin surface. These clutter signals are suppressed by reselecting the front and back peaks after an initial surface construction in a way that minimizes the range displacement between nearest neighbors, followed by a uniform smooth over the entire surface. This part of the algorithm is still being optimized with the hope that more intelligent peak-selection may result in even clearer images than those in Fig. 5. 
The results of the image processing algorithm are presented in Fig. 5(b) and (c), which show the front and back surface reconstructions. We emphasize that in these images the color scale represents range only, and not reflected power, with some additional feature enhancement coming from the global scene lighting and shading built into the Matlab 3-D graphics software used to display the data. There is no indication of the concealed gun in Fig. 5(b). Instead, the front-surface imagery faithfully reproduces the subject's shirt surface, arms, and head, including even some facial features. On the other hand, in the back-surface imagery of Fig. 5(c), the concealed weapon is plainly evident as a gun-shaped bulge on the subject's chest. The bulge is detectable because the gun thickness of about $2.5 \mathrm{~cm}$ exceeds the range resolution of the radar. Also, the gun is opaque, so that its signal returns are assigned to be part of the back surface by the imaging algorithm. If the concealed object were instead transmissive at $600 \mathrm{GHz}$, then this imaging processing algorithm would likely fail to detect it. However, more complicated signal processing based on peak anomaly detection could potentially detect concealed objects even if they were highly penetrable.

Thus unlike in imaging systems that detect total power only, including all passive imagers, the high-resolution $600 \mathrm{GHz}$ radar can separately distinguish between layers of clothing and the surface of the human body. Then, the clothing layers can be digitally "peeled away" to reveal concealed objects and weapons with high reliability. This is made possible because of a combination of the radar's huge bandwidth of nearly $30 \mathrm{GHz}$ to achieve high range resolution, its phase stability to permit coherent signal processing, and its $600 \mathrm{GHz}$ carrier frequency that readily penetrates clothing.

\section{SCALING UP: RANGE AND SPEED}

With a promising approach to detecting concealed weapons using high-resolution submillimeter-wave radar, scaling the imager's range to more useful standoff distances of $25 \mathrm{~m}$ or greater becomes a challenger of engineering rather than phenomenology. The most difficult performance tradeoff for long distance imaging is between aperture size and cross-range resolution. Unlike range resolution, which is bandwidth-limited and independent of standoff range, cross-range resolution is diffraction-limited by the radar's aperture size. The cross-range resolution scales linearly with aperture diameter, so a $25 \mathrm{~m}$ standoff image of comparable quality to the $4 \mathrm{~m}$ standoff images of Fig. 4 would require a $40 \times 25 / 4=250 \mathrm{~cm}$ diameter aperture. This large diameter is at the limit of practicability, so we estimate that standoff ranges up to $50 \mathrm{~m}$ with resolution two times worse than that of Fig. 5 is practical using the present approach.

Another standoff scaling requirement is that the radar's SNR remain high. In conventional radars that use a wide, diverging beam to detect or track a small target, the SNR scales with range as $1 / R^{4}$. This dependence is a consequence of diffraction, where the beam energy density scales as $1 / R^{2}$ over both the transmit and receive paths [5]. However, in active real-aperture imagers such as the $600 \mathrm{GHz}$ radar presented here, the outgoing beam does not diverge, but is focused to a small spot on target. The beam divergence occurs only in the receive path and so the
SNR scales as $1 / R^{2}$. This means that if a proportionally larger aperture diameter $D$ is used to maintain a fixed cross-range resolution, the resulting increase in antenna gain, which scales as $D^{2}$, will precisely compensate for the energy lost to beam divergence. Therefore a fixed target resolution guarantees that the system SNR will be independent of standoff range. A third category of system considerations for achieving longer standoff range is the speed of the backend electronics. The radar's demodulated signal frequency increases in proportion to range, requiring faster analog-to-digital conversion (ADC) and signal processing. Finally, atmospheric attenuation must be considered. Depending on atmospheric conditions (primarily humidity), the round-trip signal attenuation for $50 \mathrm{~m}$ standoff is expected to lie anywhere between $1 \mathrm{~dB}$ and $50 \mathrm{~dB}$ at $600 \mathrm{GHz}$ [1], but at the local attenuation minimum of $670 \mathrm{GHz}$, the worst case scenario is only a $20 \mathrm{~dB}$ loss.

We have not completed a systematic experimental investigation of these range-scaling effects for the $600 \mathrm{GHz}$ imaging radar. However, preliminary measurements at $25 \mathrm{~m}$ standoff indicate that through-garment weapons detection will be viable at this long range, and that signal loss from atmospheric absorption may be surprisingly low. The $25 \mathrm{~m}$ standoff measurements were made using an off-axis reflector similar to that shown in Fig. 2, but with a diameter of $47 \mathrm{~cm}$ rather than $40 \mathrm{~cm}$. Owing to the much larger range and only slightly larger aperture, the resulting image resolution is expected to be more than five times worse than the $4 \mathrm{~m}$ standoff images of Fig. 5 . The $3 \mathrm{~mm}$ bead calibration at $25 \mathrm{~m}$ standoff confirmed that the two-way beam width is significantly degraded, with additional distortion (likely from reflector aberrations or optical misalignment) yielding a 3 $\mathrm{dB}$ beam size of about $2 \times 3.4 \mathrm{~cm}$.

Yet despite this inferior resolution at $25 \mathrm{~m}$ standoff, the results in Fig. 6 indicate that through-garment imaging is still effective even at this range. Fig. 6(a) shows a photograph of the scenario, where a mannequin's torso has a 2.25 in steel pipe hung around its neck. A potential pitfall of using a plastic mannequin is that it is highly transmissive at $600 \mathrm{GHz}$, while humans are totally opaque. Thus the mannequin was covered with a damp cotton shirt (water is extremely absorbent at $600 \mathrm{GHz}$ ), which in turn was covered by plastic wrap to prevent the outer clothing from becoming moist. During the scan the pipe lay underneath a loose-fitting T-shirt, as shown in Fig. 6(b).

To compensate for the lowered SNR resulting from an increase in the standoff-to-aperture ratio, the radar chirp time was increased to $125 \mathrm{~ms}$. The FMCW radar span was maintained at $28.8 \mathrm{GHz}$. The resulting $81 \times 73$ pixel images of the 3-D frontand back-surface reconstructions are shown in Fig. 6(c) and (d). Because the steel pipe is opaque at $600 \mathrm{GHz}$, the usual image processing algorithm makes the pipes visible as an unnatural protrusion in the torso in Fig. 6(d).

The clarity of the pipe, however, is not very good because of the distorted beam shape and the difficulty of extracting the correct range-compressed peaks corresponding to the front and back surfaces when the large beam encounters a target with a complex topography. We expect that the $25 \mathrm{~m}$ image quality will improve rapidly with increasing aperture size, but these results prove that the $600 \mathrm{GHz}$ imaging radar is still effective at exposing concealed objects even at $25 \mathrm{~m}$ standoff. 

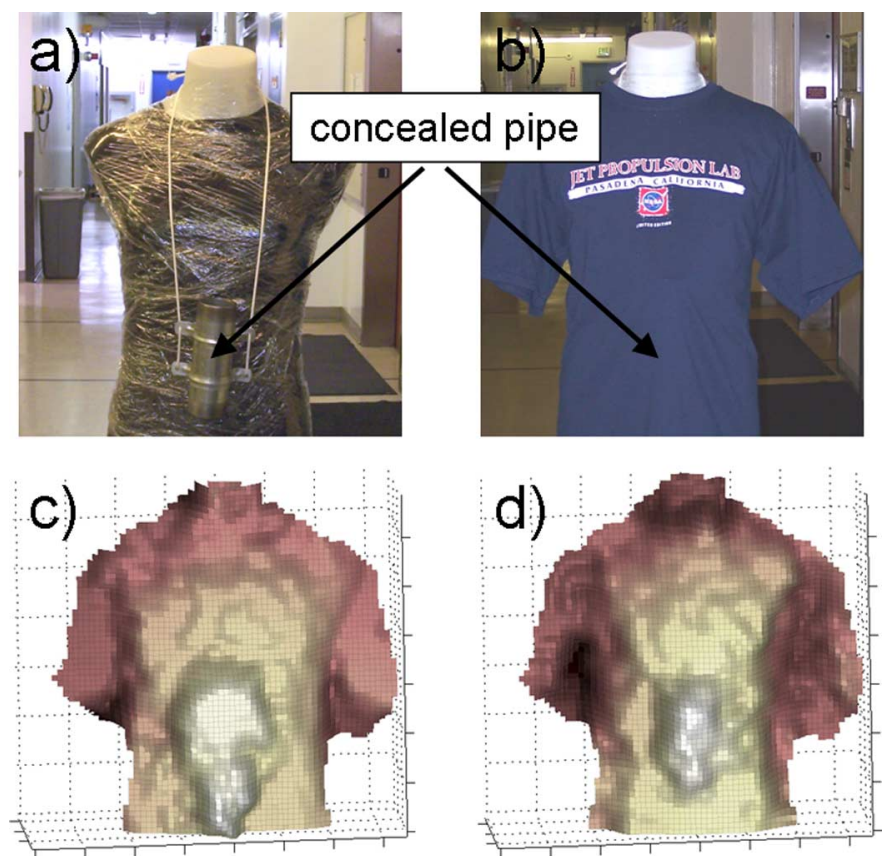

Fig. 6. (a) Target scenario is a plastic mannequin wrapped in damp fabric and plastic at $25 \mathrm{~m}$ standoff range. A 2.25 in steel pipe was hung around the neck. (b) During the scan, a T-shirt was placed over the mannequin and pipe. (c) and (d) Front- and back-surface reconstructions revealing the presence of the pipe. The color scale represents range only.

As a second test at $25 \mathrm{~m}$ standoff, we measured the effect of rain on the reflected signal intensity of the $600 \mathrm{GHz}$ radar. During a rainstorm that occurred at the Jet Propulsion Laboratory on December 7, 2007, the radar beam was directed out of our building's loading dock, and the reflection was measured from the same $3 \mathrm{~mm}$ gold bead target shown in Fig. 2(b). The beam traveled $2.5 \mathrm{~m}$ indoors before leaving the building, with a round-trip path through the rain of about $45 \mathrm{~m}$. Data obtained through the rain during a particularly heavy downpour, when the JPL weather station reported $92 \%$ relative humidity at a temperature of $12.6^{\circ} \mathrm{C}$, are shown in Fig. 7. The figure also shows the radar signal from the same target (at $25 \mathrm{~m}$ range) obtained in a hallway entirely indoors. In both cases the gold bead signal is well above the noise floor, and the heavy rain caused an attenuation of only $7 \mathrm{~dB}$, or about $150 \mathrm{~dB} / \mathrm{km}$. This is consistent with the $600 \mathrm{~dB} / \mathrm{km}$ attenuation calculated for $90 \%$ relative humidity at $35^{\circ} \mathrm{C}$ in [1], since the saturated absolute water content in air at that temperature is about four times higher than at the temperature during our measurement. The measurements of Fig. 6 suggest that images obtained at a standoff range of $25 \mathrm{~m}$ will not be severely degraded by atmospheric attenuation in most scenarios with our current SNR.

Just as increasing the standoff range of the radar will be necessary in a fielded system, so will shortening the image acquisition time. For a single-pixel instrument a speed improvement of 2-3 orders of magnitude is needed to achieve more than one frame per second. One attractive approach for this is to construct a linear array of sensors that rapidly scans in a single direction. This strategy has been successfully exploited in the passive millimeter wave imager developed by Sago Systems. ${ }^{1}$ Building a

\footnotetext{
${ }^{1}$ Online]. Available: www.sagosystems.com
}

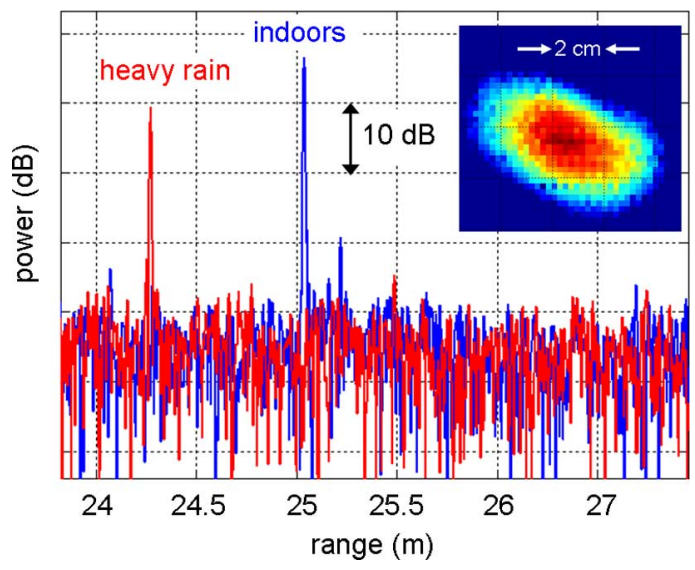

Fig. 7. Comparison of the range-compressed radar returns from a $3 \mathrm{~mm}$ gold bead at $25 \mathrm{~m}$ standoff, taken in conditions of heavy rain and indoors. Attenuation from rain is only $7 \mathrm{~dB}$. Inset: Beam profile of bead at $25 \mathrm{~m}$ standoff. The color scale spans $10 \mathrm{~dB}$ of dynamic range, and the beam shape distortion is likely from aberrations in the aperture shape.

linear array of receivers or transceivers for the imaging radar would require a re-design of the front-end submillimeter components and the associated optics. However, in contrast to systems that generate terahertz radiation using laser downconversion or vacuum tubes, such an array is feasible because the all-solid-state Schottky diode devices we employ can be made extremely compact using integrated circuit and microfabrication techniques. Finally, the signal processing load of an imaging radar array also increases with pixel number and speed. For a linear array operating at a $1 \mathrm{kHz}$ pulse repetition frequency, the IF signal frequencies would still only be a few $\mathrm{MHz}$, and so the processing requirements of the range-compressed spectra are modest enough to be handled in real time by a dedicated processor.

\section{CONCLUSION}

We have shown that a $600 \mathrm{GHz}$ FMCW radar system can be used to detect weapons or contraband under clothing at significant stand-off distances, and that simple active imaging using a CW source reflected off a target cannot easily distinguish between scene clutter and hidden objects due to variations in object backscatter. By employing an FMCW radar with high range resolution, a front- and rear-surface detection algorithm can reveal hidden objects with high resolution. This added capability would make a critical difference to a real-world deployable instrument.

Although some of the required RF hardware is not available off-the-shelf at this time, we know from our own development efforts that adequate room temperature solid-state sources and heterodyne sensors can be designed to meet the requirements of such a system. Extending the effective range for a given lateral resolution is a practical issue driven mainly by aperture scale. Fortunately, in the active radar imaging approach the backscattered signal strength does not degrade with distance if the lateral resolution is maintained, and range resolution is also independent of standoff range. Atmospheric attenuation can be a concern, but working in an atmospheric window (such as $670 \mathrm{GHz}$ ) 
mitigates this problem. As we have already shown, measurements even at $600 \mathrm{GHz}$ under realistic conditions in a driving rainstorm produced only a $7 \mathrm{~dB}$ loss in SNR at $25 \mathrm{~m}$. The biggest hurdle, we believe, is in bringing the imaging system up to video rate. However this should be possible by employing a linear transceiver array, and we are currently pursuing this avenue of research.

\section{ACKNOWLEDGMENT}

The authors wish to thank Dr. J. Ward of the Jet Propulsion Laboratory (JPL), Pasadena, CA, for providing the $600 \mathrm{GHz}$ sources.

\section{REFERENCES}

[1] R. Appleby and H. B. Wallace, "Standoff detection of weapons and contraband in the $100 \mathrm{GHz}$ to $1 \mathrm{THz}$ region," IEEE Trans. Antennas Propag., vol. 55, no. 11, pp. 2944-2956, Nov. 2007.

[2] E. Schlecht, J. Gill, R. Dengler, R. Lin, R. Tsang, and I. Mehdi, "First wideband 520-590 GHz novel balanced fundamental Schottky mixer," in 18th Int. Space Terahertz Technol. Symp., Pasadena, CA, 2007, p. 296.

[3] E. Schlecht, J. Gill, R. Dengler, R. Lin, R. Tsang, and I. Mehdi, "A unique 520-590 GHz biased subharmonically-pumped Schottky mixer," IEEE Microw. Wireless Compon. Lett., vol. 17, no. 12, pp. 879-881, Dec. 2007.

[4] A. Maestrini, J. S. Ward, J. J. Gill, H. S. Javadi, E. Schlecht, C. TriponCanseliet, G. Chattopadhyay, and I. Mehdi, "A 540-640-GHz highefficiency four-anode frequency tripler," IEEE Trans. Microw. Theory Tech., vol. 53, no. 9, pp. 2835-2843, Sep. 2005.

[5] M. Skolnik, Introduction to Radar Systems. Boston, MA: McGrawHill, 2001.

[6] K. B. Cooper, R. J. Dengler, G. Chattopadhyay, E. Schlecht, J. Gill, A. Skalare, I. Mehdi, and P. H. Siegel, "A high-resolution imaging radar at $580 \mathrm{GHz}$," IEEE Microw. Wireless Compon. Lett., vol. 18, no. 1, pp. 64-66, Jan. 2008.

[7] R. J. Dengler, K. B. Cooper, G. Chattopadhyay, I. Mehdi, E. Schlecht, A. Skalare, C. Chen, and P. H. Siegel, "600 GHz imaging radar with $2 \mathrm{~cm}$ range resolution," in IEEE MTT-S Int. Microw. Symp. Dig., Honolulu, HI, Jun. 2007, pp. 1371-1374.

[8] D. J. Benford, M. C. Gaidis, and J. W. Kooi, "Optical properties of Zitex in the infrared to submillimeter," Appl. Opt., vol. 42, no. 25, pp. 5118-5122, Sep. 2003.

[9] E. L. Jacobs, S. Moyer, C. C. Franck, F. C. DeLucia, C. Casto, D. T. Petkie, S. R. Murrill, and C. E. Halford, "Concealed weapon identification using terahertz imaging sensors," Proc. SPIE, vol. 6212, pp. 62120J-1, 2006

Ken B. Cooper (M'06) received the A.B. degree in physics (summa cum laude) from Harvard College, Cambridge, MA, in 1997, and the Ph.D. degree in physics from the California Institute of Technology, Pasadena, in 2003.

Since 2006, he has been a Member of the Technical Staff with the Jet Propulsion Laboratory, Pasadena, CA. His current research interests include submillimeter-wave radar, spectroscopy, and device physics.

Robert J. Dengler (M'04) was born in Whittier, CA in July 1963. He received the Bachelor of Science degree in electrical and computer engineering from the California State Polytechnic University, Pomona, in 1989.

He began his work with the Jet Propulsion Laboratory as an Intern in 1988, where he developed beam pattern acquisition and analysis software. Since then he has been involved in the design and construction of submillimeter-wave receivers and components, including design and fabrication of test instrumentation for submillimeter flight mixers. His recent reserach has focused on submillimeter and teranertz active and passive imaging, including design and construction of a very high dynamic range small-sample transmission imaging system operating at $2.5 \mathrm{THz}$.
Nuria Llombart (S'06-M’07) received the Ingeniero de Telecomunicación degree and Ph.D. degree from the Universidad Politécnica de Valencia, Spain, in 2002 and 2006, respectively.

From 2002 to 2007, she was with the Defence, Security and Safety Institute of the Netherlands Organization of Applied Scientific Research (TNO), The Hague, The Netherlands. She is currently a Postdoctoral Fellow with the California Institute of Technology, Pasadena, working for the Submillimeter-Wave Advanced Technology (SWAT) Group, Jet Propulsion Laboratory. Her research interests include the analysis and design of printed array antennas, EBG structures, reflector antennas and submillimeter-wave components.

Dr. Llombart was a corecipient of the 2008 H. A. Wheeler Applications Prize Paper Award from the IEEE Antennas and Propagation Society.

Tomas Bryllert received the M.S. degree in physics and Ph.D. degree in semiconductor physics from Lund University, Lund, Sweden, in 2000 and 2005, respectively.

In 2006, he joined the Physical Electronics Laboratory, Chalmers University of Technology, Göteborg, Sweden, where his main research interest was device and circuit technology for terahertz frequency multipliers. HE is currently with the Jet Propulsion Laboratory (JPL), Pasadena, CA, funded by a research fellowship from the Wallenberg Foundation. He is involved with submillimeter-wave imaging radar and terahertz time-domain imaging systems.

Goutam Chattopadhyay (S'93-M'99-SM'01) received the B.E. degree in electronics and telecommunication engineering from the Bengal Engineering College, Calcutta University, Calcutta, India, in 1987, the M.S. degree in electrical engineering from the University of Virginia, Charlottesville, in 1994, and the Ph.D. degree in electrical engineering from the California Institute of Technology, Pasadena, in 1999.

He is currently a Senior Member of the Technical Staff with the Jet Propulsion Laboratory (JPL), Pasadena, CA. His research interests include microwave, millimeter-, and submillimeter-wave heterodyne and direct detector receivers, frequency sources and mixers in the terahertz region, antennas, SIS mixer technology, and direct detector bolometer instruments.

Erich Schlecht (S'94-M'95) received the B.A. degree in astronomy and physics and M.S. degree in engineering physics from the University of Virginia, Charlottesville, in 1981 and 1987, respectively, and the Ph.D. degree in electrical and computer engineering from The Johns Hopkins University, Baltimore, MD in 1999.

In November 1998, he joined the engineering staff of the Jet Propulsion Laboratory (JPL), Pasadena, CA, as a member of the Submillimeter-Wave Advanced Technology (SWAT) Group. He performs circuit design and Schottky diode modeling for sub-millimeter and terahertz $\mathrm{LO}$ frequency multipliers and mixers.

John Gill received the B.S. and M.S. degrees in mechanical engineering from the University of California at Los Angeles in 1997, and the Ph.D. degree in microelectromechanical systems (MEMS) from University of California at Los Angeles in 2001.

From 1997 to 1998, he was with the Jet Propulsion Laboratory (JPL), Pasadena, CA, where he was involved in developing the quantum-well infrared photodetector. He returned to the JPL, where he currently develops microwave devices. His research interests include design, fabrication and characterization of microelectronic devices using MEMS and nanoelectromechanical systems (NEMS) technologies for space and biomedical applications.

Choonsup Lee received the B.S. degree in electrical engineering from Kyungpook National University, Daegu, Korea, in 1996, and the M.S. and Ph.D. degrees in electrical engineering and computer science from the Korea Advanced Institute of Science and Technology (KAIST), Daejeon, Korea, in 1998 and 2002, respectively.

He is currently a Member of the Technical Staff with the Jet Propulsion Laboratory (JPL), Pasadena, CA. He has extensive experiences in the design and characterization of MEMS/NEMS devices. He is currently involved with GaAsbased frequency sources and mixers in the terahertz region. He has authored or coauthored 17 international journal papers and 29 international conference papers. 
Anders Skalare (S'88-M'90), photograph and biography not available at time of publication.

Imran Mehdi (S'83-M'90-SM'05) received the B.S.E.E., M.S.E.E., and Ph.D. degrees from The University of Michigan at Ann Arbor, in 1985, 1986 and 1990, respectively.

He is a Principal Member of Engineering staff with the Jet Propulsion Laboratory (JPL), California Institute of Technology, Pasadena, where his responsibilities include developing terahretz components and subsystems for current and future NASA missions. He led the development of low-parasitic planar Schottky diodes for submillimeter-wave applications. These devices have now been implemented on missions such as the Microwave Limb Sounder (ozone monitoring), MIRO (comet rendezvous), and HIFI (astrophysics). His current interests include millimeter and submillimeter-wave devices, high-frequency instrumentation, and technologies for miniaturizing submillimeter-wave heterodyne receiver systems.

Peter H. Siegel (S'77-M'83-SM'98-F'01) received the B.A. degree in astronomy from Colgate University, Hamilton, NY, in 1976, and the M.S. degree in physics and $\mathrm{Ph} . \mathrm{D}$. degree in electrical engineering from Columbia University, New York, NY, in 1978 and 1983 respectively.
He served as an NRC Fellow with the Goddard Institute for Space Studies, New York, NY, and then as a staff member with the Electronics Development Laboratory, National Radio Astronomy Observatory, Charlottesville, VA, until 1987. He then joined the Jet Propulsion Laboratory (JPL), Pasadena, CA, to begin work on submillimeter-wave sensors for NASA space astrophysics and remote sensing applications. While with the JPL, he has been involved in four space flight missions and many dozens of research and development programs. He founded and has led for more than 15 years a large technical team, Submillimeter Wave Advanced Technology (SWAT), which is focused on NASA applications of terahertz technology. In 2001, he joined the staff of the Division of Biology, California Institute of Technology, where he currently holds a joint appointment. With the California Institute of Technology, he has expanded his terahertz applications into biology and medicine. In 2007, he became a faculty associate in electrical engineering where he continues to broaden terahertz activities into cross-disciplinary areas. His interests span all areas of terahertz technology and applications from space science to biology.

Dr. Siegel is an active member of the terahertz community and has served as chair of MTT 4, THz Technology. He has been an IEEE Distinguished Lecturer in terahertz technology and applications. He is currently chair of the organizing committee of the International Conference on Infrared, Millimeter, and Terahertz Waves, the largest continuous forum devoted to terahertz science and technology. 